\title{
Multicarrier-Based QAPM Modulation System for the Low Power Consumption and High Data Rates
}

\author{
Jae-Hoon Choi • Heung-Gyoon Ryu • Xuedong Liang
}

Published online: 5 April 2011

(C) The Author(s) 2011. This article is published with open access at Springerlink.com

\begin{abstract}
Low power consumption and high data rate are the most important requirements for the communication system. Especially, very low power consumption modulation method is required for the short range communication systems such as the medical implantable communication devices or capsule endoscope, and so on. For the higher data rate, we like to combine the OFDM system into the QAPM since the OFDM system has higher bandwidth efficiency than a single-carrier system. In this paper, we like to propose a QAPM (Quadrature amplitude position modulation) method combined with the orthogonal frequency-division multiplexing (OFDM) system. Next, we analyze the performance of three low-powerconsumption modulation schemes: the phase shift position modulation (PSPM), phase silence shift keying (PSSK), and QAPM using orthogonal frequency-division multiplexing (OFDM) system in the multi-path channel. These schemes have lower bandwidth efficiency and the higher power efficiency than the existing phase-shift keying (PSK) and quadrature amplitude modulation (QAM) schemes. It can be shown that they can achieve greater power efficiency because every modulation symbol has a zero-
\end{abstract}

\footnotetext{
J.-H. Choi $\cdot$ H.-G. Ryu $(\bowtie)$

Department of Electronic Engineering,

Chungbuk National University,

361-763, Cheongju, Korea

e-mail: ecomm@cbu.ac.kr

J.-H. Choi

e-mail: arcien@cbnu.ac.kr

X. Liang

Department of Electronic and Computer Engineering,

University of British Columbia,

Vancouver, Canada

e-mail: xuedongl@ece.ubc.ca
}

envelope period as in pulse-position modulation (PPM) techniques. Finally, we compare the performances of the PSPM, PSSK, and QAPM modulation combined with the OFDM system with regard to bit error rate performance and throughput.

Keywords PSPM · PSSK - QAPM · power efficiency · bandwidth efficiency $\cdot$ throughput $\cdot$ WBAN

\section{Introduction}

With recent growth in the use of portable communication devices and networked sensor systems, communication systems that consume little power have become more important. From among these, WPAN and WBAN are actively studies. The WPAN systems are composed of short range high data rate communication system such as UWB and a sensor network system such as Bluetooth and Zigbee. A UWB system is required low transmitted power for in order to reduce interference with other wireless devices. Also, the sensor network systems are required low power consumption for the running time. The WBAN systems are composed of in-body and on-body systems. And the inbody applications which interconnect the implanted apparatus in the human body and the apparatus sticking on the human body support a wide range of medical applications [1]. Therefore WBAN devices require a high degree of miniaturization and long operation time periods before maintenance. Because the system with small sized battery inside the body and the battery is not easy to change.

Studies of low-power-consumption communication systems are based on the frequency-shift keying (FSK), pulseposition modulation (PPM), and pulse-width modulation (PWM) schemes $[2,3]$. These schemes are more power 
Fig. 1 PSPM constellation

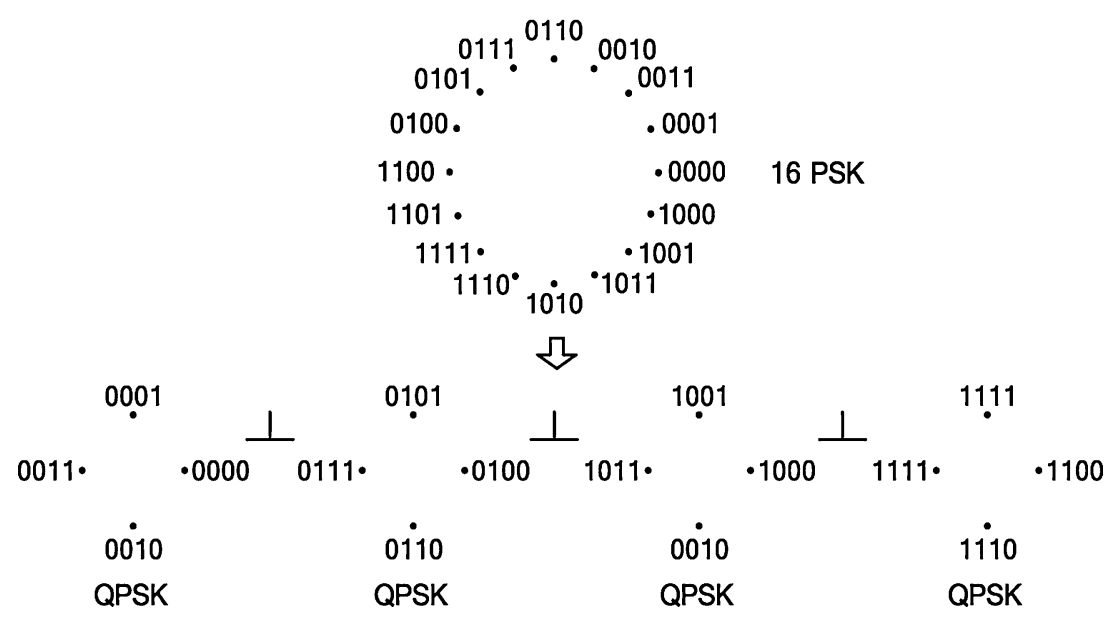

efficient than phase-shift keying (PSK) and Quadrature amplitude modulation (QAM), but their bandwidth efficiency is very poor. For this reason, they require a high bandwidth.

The phase silence shift keying (PSSK) modulation scheme was proposed to address these problems with existing modulation schemes [4]. It has better bandwidth efficiency than FSK and better power efficiency than PSK. These characteristics are suitable for capsule-endoscopes, which require a communication system with low power consumption and a high data rate [5]. The PSSK modulation scheme determines the symbol location by using the first bit data in a symbol period. Therefore, this modulation scheme transmits both symbols and zeros, like PPM.

The PSPM modulation scheme is an extension of the PSSK technique. The bandwidth efficiency of PSPM is half that of PSSK, but its bit error rate (BER) performance is more than $5 \mathrm{~dB}$ better than that of PSSK. Also, PSPM is more power efficient than PSK and PSSK because the modulation level is lower. This modulation scheme transmits four PSK symbols of an orthogonal waveform. This modulation uses two bits to determine the absence marking the position of the symbol's silence envelope. This characteristic is suitable for applications requiring low power consumption.

The QAPM modulation scheme is an extension of the QAM technique, unlike PSSK and PSPM [6, 7]. This method requires twice the bandwidth of QAM but the same bandwidth as PSSK. However, its BER performance is 2
$4 \mathrm{~dB}$ better than that of QAM. Also, the power efficiency of the transmitter is improved by $3 \mathrm{~dB}$.

In this paper, we propose a new QAPM method combined with OFDM and analyze to compare the performance of low-power-consumption modulation schemes such as PSPM and PSSK using OFDM transmission in the multi-path channel. And this paper is organized as follow. Section 2 describes the conventional method. And Section 3 describes the proposed QAPM method combined with the orthogonal frequency division multiplexing. Section 4 presents the multi-path channel model. Finally, the simulation results are given in Section 5.

\section{PSPM and PSPM modulation}

\subsection{PSPM modulation scheme}

Figure 1 is 16PSPM constellation. The existing PSSK method transmit 16PSSK modulation by using two orthogonal 8PSSK symbol, but 16PSPM modulation by using four orthogonal QPSK symbol. This method is two bit determines the silence-envelope position of the symbol, and $\left(\log _{2} M\right)-2$ bits determine the phase of the symbol. Therefore this method combine two bit PPM and $\left(\log _{2} M\right)-2$ bit PSK.

Figure 2 shows symbol duration of PSPM. The symbol period of PSPM is decrease than existing PSSK, but bandwidth is increase than existing PSSK.

Fig. 2 PSPM symbol duration

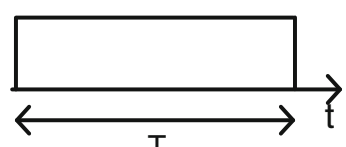

(a) ${ }^{\mathrm{P}} \mathrm{PSK}$

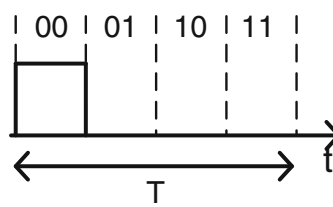

(b) PSPM

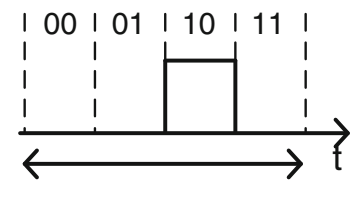




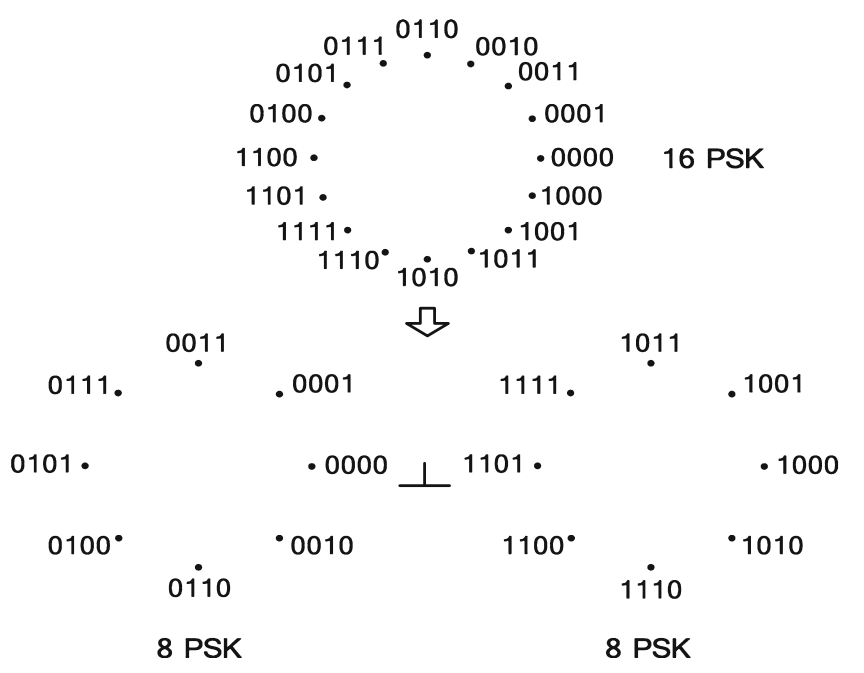

Fig. 3 16PSSK constellation

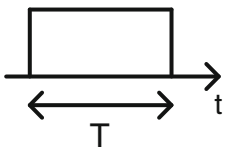

(a) PSK

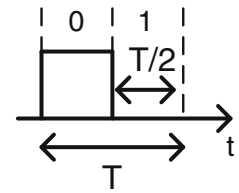

(b) PSSK

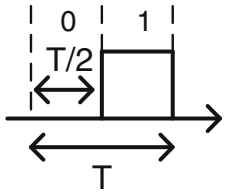

$\mathrm{T}$

Fig. 4 16PSSK symbol duration

Fig. 5 OFDM based QAPM system model
The M-ary PSPM for $M \geq 8$ is represented as follows.

$$
\begin{aligned}
& D_{m}(t)=\sum_{n=0}^{3} A_{m, n} \alpha(t-n T / 4) \exp \left[j \theta_{m}\right](0 \leq t \leq T), \\
& A_{m, n}=\left\{\begin{array}{l}
1, n<\text { floor }(4 m / M)<n+1 \\
0, \text { otherwise }
\end{array}\right.
\end{aligned}
$$

$\alpha_{n}(t)=u[t-0.25(n-1) T]-u[t-0.25 n T], n=1,2,3,4$

Here, $\alpha_{n}(t)$ is a unit step function that determines the position of a symbol. If the $\alpha_{n}(t)$ is zero, transmitted signal $\mathrm{D}_{m}(t)$ is zero. But, if the $\alpha_{n}(t)$ is one, transmitted signal $\mathrm{D}_{m}(t)$ is $e^{j \theta_{m}}$. Therefore, the transmitted signal $\mathrm{s}_{m}(t)$ can be written using Eq. 1, which retains the orthogonality of the symbol by $A_{m, n}$.

$s_{m}(t)=\sum_{n=0}^{3} A_{m, n} \alpha(t-n T / 4) e^{j \theta_{m}}$

\subsection{PSSK modulation scheme}

PSSK modulation scheme is transmitting two PSK symbol of orthogonal waveform. As shown Fig. 3, the 16PSSK constellation. The 16PSK is transmitting 4-ary data of one phase symbol, but 16PSSK transmitting 4-ary data of two 8PSK orthogonal symbols. Thus PSSK is one bit determines the silence-envelope position of the symbol, and $\left(\log _{2} M\right)-1$ bits determine the phase of the symbol. As shown Fig. 4, the PSK and PSSK symbol duration. In Fig. 4(a), PSK symbol duration is $T$ which is symbol period, But PSSK symbol duration is $T / 2$ as Fig. 4(b). The symbol position of PSSK determines using by the first bit data. This method is double portion of bandwidth more than that of PSK, but between the

system model
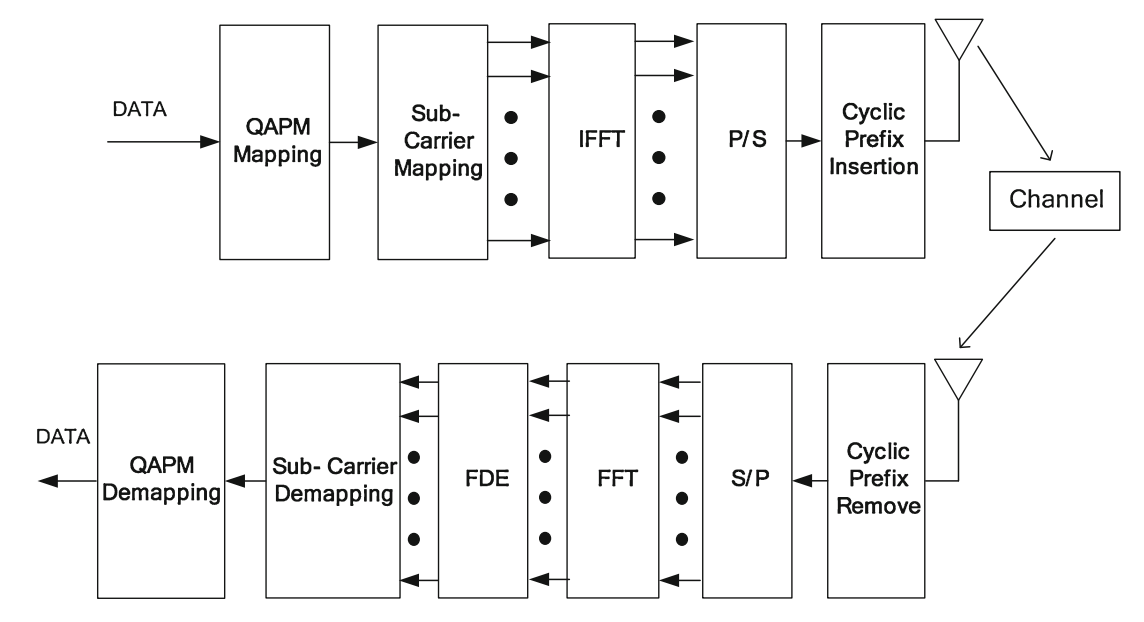
QAPM symbol

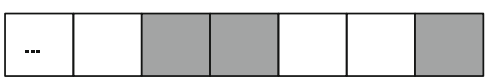

Zero symbol

Sub-carrier

Mapping

QAM symbol

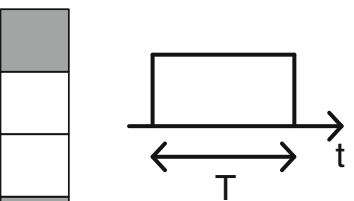

(a) QAM
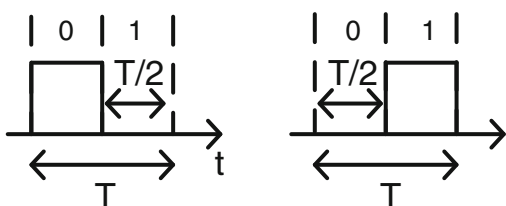

(b) QAPM

Fig. 8 16QAPM symbol duration

$s_{m}(t)=A_{m}\left(\cos \theta_{m}+A_{m} \sin \theta_{m}\right) \alpha(t)+B_{m}\left(\cos \theta_{m}+\sin \theta_{m}\right) \beta(t)$

Fig. 6 QAPM subcarrier mapping

symbols secure to hamming distance because M-ary PSSK is transmitting $\mathrm{M} / 2$-ary PSK.

The M-ary PSSK signal is represented as follows.

$s_{m}(t)=\operatorname{Re}\left\{\left(A_{m} \alpha(t)+B_{m} \beta(t)\right) \exp \left[j \theta_{m}\right]\right\}(0 \leq t \leq T)$

Here, $\theta_{m}=2 \pi \bmod (m, 0.5 M) / 0.5 M ; \operatorname{MOD}(a, b)$ is the modulus after $a$ is divided by $b$, and $T$ is the symbol period. Therefore, the phase $\theta$ varies as $4 \pi / M$.

$A_{m}= \begin{cases}1, & 0 \leq m \leq \frac{M}{2}-1 \\ 0, & \frac{M}{2} \leq m \leq M-1\end{cases}$

$\alpha(t)=u(t)-u(t-0.5 T), \beta(t)=\alpha(t-0.5 T)$

$A_{m}$ and $B_{m}$ are the position of the symbol in the symbol period, and $\alpha(t)$ and $\beta(t)$ are unit step functions that determine the carrier frequency of the symbol.
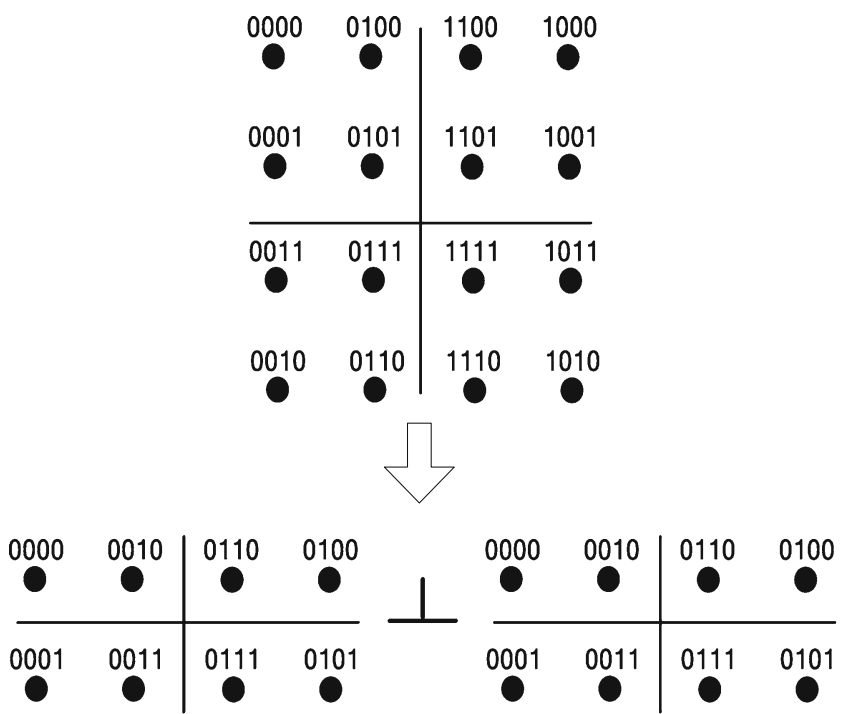

Fig. 7 16QAPM constellation

Therefore, the transmitted signal $s_{m}(t)$ can be written using Eq. 8, which retains the orthogonality of the symbol by $A_{m}$ and $B_{m}$.

\section{Proposed system model}

\subsection{OFDM system model}

The Fig. 5 is block diagram of proposed system model. QAPM-OFDM transmission system is using basic OFDM system. This system is transmitting through IFFT after QAPM subcarrier mapping.

Subcarrier mapping was shown in Fig. 6. A QAPM symbol consists of QAM symbol and zero symbols. Therefore subcarrier was arranged as shown Fig. 6.

\subsection{QAPM modulation scheme}

QAPM modulation scheme is transmitting two QAM symbol of orthogonal waveform. As shown in Fig. 7, 16QAPM constellation. 16QAM is transmitting 4-ary data of one symbol, but 16QAPM transmitting 4-ary data of two 8QAM orthogonal symbols.

In Fig. 8(a), QAM symbol duration is $T$ which is symbol period, but QAPM symbol duration is $T / 2$ as Fig. $8(\mathrm{~b})$. The symbol position of QAPM determines using the first bit data. This method is double portion of bandwidth more than QAM.

The M-ary QAPM modulation is represented as follows.

$D_{m}(t)=\left\{\left(A_{I}+A_{J}\right) A_{m} \alpha(t)+\left(A_{I}+A_{J}\right) B_{m} \beta(t)\right\}(0 \leq t<T)$

$A_{m}= \begin{cases}1, & 0 \leq m \leq \frac{M}{2}-1 \\ 0, & \frac{M}{2} \leq m \leq M-1\end{cases}$

$\alpha(t)=u(t)-u(t-0.5 T), \beta(t)=\alpha(t-0.5 T)$ 


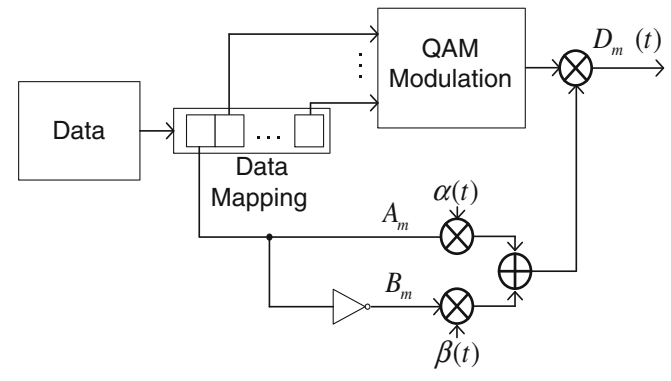

(a) QAPM demodulation

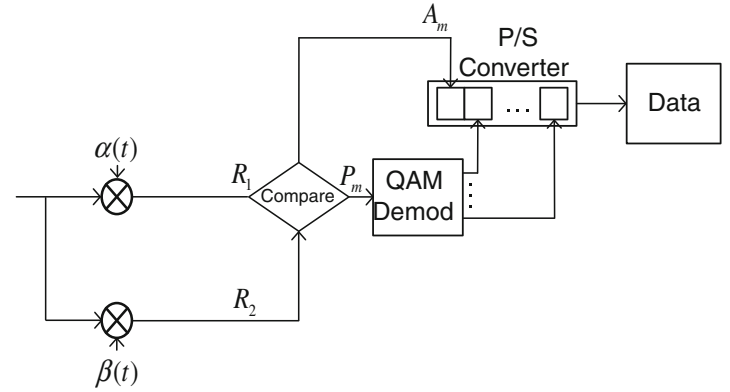

(b) QAPM demodulation.

Fig. 9 QAPM modulation and demodulation

Here, $A_{I}$ is the in-phase amplitude, and $A_{J}$ is the quadrature amplitude. $A_{m}$ and $B_{m}$ are the position data of the symbol in symbol period.

As shown in Fig. 9(a), QAPM modulation scheme will be process in 3-step. At first, the input data is mapped into QAPM data as Fig. 6. Next, this QAPM mapping data is modulated the QAM data. Finally, a modulation data is modulated the PPM using $A_{m}$ and $B_{m}$ as Fig. 3.

The Fig. 9(b) shows the QAPM demodulation scheme. The QAPM demodulation scheme will be progress 3-step as same modulation scheme.

$$
\begin{aligned}
& R_{1}(t)=\left(D_{m}(t)+n(t)\right) \times \alpha(t)=A_{m}\left(A_{I}+A_{J}\right)+n(t) \alpha(t) \\
& R_{2}(t)=\left(D_{m}(t)+n(t)\right) \times \beta(t)=B_{m}\left(A_{I}+A_{J}\right)+n(t) \beta(t)
\end{aligned}
$$

At first, we can detect the symbol position in period. The signal power compare $R_{1}$ and $R_{2}$ that we use to detect symbol position.

$$
\max \left(\left|R_{n}\right|^{2}\right)= \begin{cases}R_{1} \Rightarrow & A_{m}=0, P_{m}=r_{1}+j \cdot r_{2} \\ R_{2} \Rightarrow & A_{m}=1, P_{m}=r_{3}+j \cdot r_{4}\end{cases}
$$

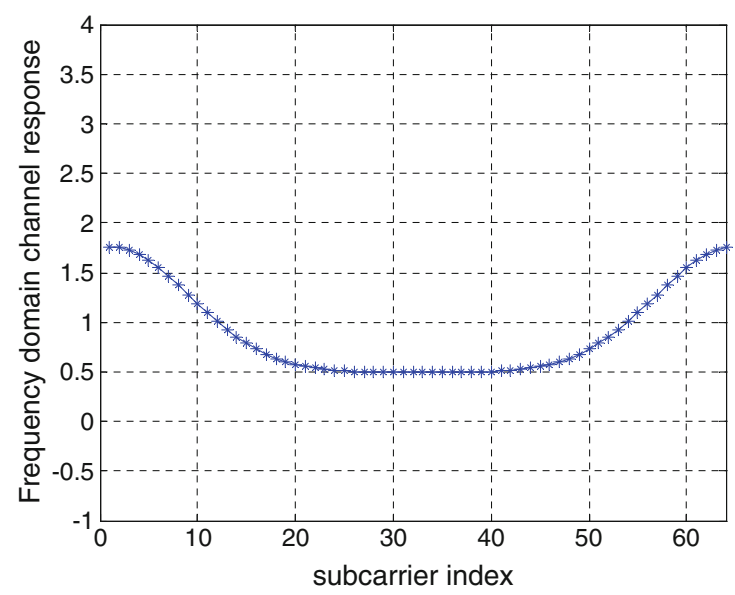

(a) Pedestrian A model.
If is $R_{1}>R_{2}$, than $A_{m}$ is 0 . And if is $R_{1}<R_{2}$, than $A_{m}$ is 1 . Therefore QAPM signal be able to demodulation by sum $A_{m}$ and QAM demodulation data. M-ary QAPM is one bit determines the silence-envelope position of the symbol, and $\left(\log _{2} M\right)$ bits QAM symbol.

\section{Simulation results}

Figure 10 shows the multi-path channel model used in the simulation [8]. Figure 10(a) shows the Pedestrian A channel model, which shows moderate fading and a short delay time. Figure 10(b) shows the Vehicular A model, which has a long delay time and severe fading. We used this channel model in the simulations. We also used a channelequalizing method such zero forcing. Table 1 shows the delay profiles of these models.

Table 2 describes the OFDM simulation environment. We conducted the simulations according to the IEEE 802.11a standard. Also, we transmitted 250,000 OFDM symbols for calculating the throughput.

Figure 11 shows the simulated low-power-consumption modulation in the AWGN channel. As shown in Fig. 11(a),

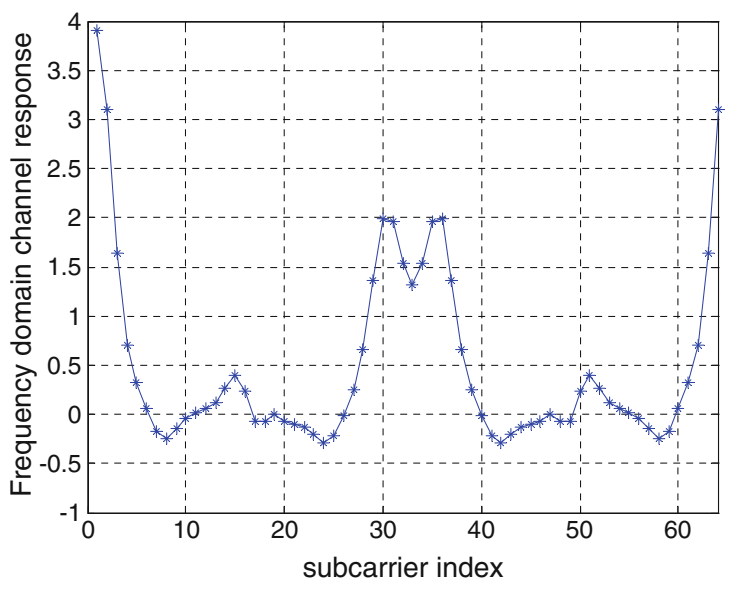

(b) Vehicular A model.

Fig. 10 Frequency response of multi-path channel model 
Table 1 Delay profile of multi-path channel

\begin{tabular}{|c|c|c|c|c|}
\hline \multirow[t]{2}{*}{ Tap } & \multicolumn{2}{|c|}{ Pedestrian A } & \multicolumn{2}{|l|}{ Vehicular A } \\
\hline & $\begin{array}{l}\text { Relative } \\
\text { delay (ns) }\end{array}$ & $\begin{array}{l}\text { Average } \\
\text { power }(\mathrm{dB})\end{array}$ & $\begin{array}{l}\text { Relative } \\
\text { delay (ns) }\end{array}$ & $\begin{array}{l}\text { Average } \\
\text { power }(\mathrm{dB})\end{array}$ \\
\hline 1 & 0 & 0 & 0 & 0 \\
\hline 2 & 110 & -9.7 & 310 & -1.0 \\
\hline 3 & 190 & -19.2 & 710 & -9.0 \\
\hline 4 & 410 & -22.8 & 1090 & -10.0 \\
\hline 5 & & & 1730 & -15.0 \\
\hline 6 & & & 2510 & -20.0 \\
\hline
\end{tabular}

at a BER of $10^{-6}$, the BER performance a 16-ary PSPM is more than $5.6 \mathrm{~dB}$ better than that of a 16-ary PSSK, and the BER performance of a 16-ary PSSK is more than $6 \mathrm{~dB}$ better than that of PSK. The BER performance of a 16-ary QAPM is better than that of a 16-ary PSSK. These results are consistent with the simulation results for a single-carrier system. Likewise, Fig. 11(b) shows that 32-ary simulation results are the same as those of a single-carrier system. These results show that PSPM modulation has the best BER performance.

Table 3 described the power efficiency and bandwidth efficiency of modulations in base-band. As shown in Table 3, the bandwidth efficiency of PSPM is half than PSSK and QAPM because PSPM requires double portion of bandwidth more than that of PSSK. Also, the bandwidth efficiency of PSSK and QAPM is half than PSK and QAM. But, BER performance of PSPM is better than other modulation scheme. The duty gain is means symbol period to pulse-on duration. Therefore duty gain of PSPM is $6 \mathrm{~dB}$, duty gain of PSSK and QAPM are $3 \mathrm{~dB}$.

Figure 12 shows the simulated BER performance of the low-power-consumption modulation schemes in the multi-

Table 2 Simulation environment

\begin{tabular}{ll}
\hline FFT size & 64 \\
OFDM Sub-carrier & 52 \\
Data Sub-carrier & 48 \\
Pilot carrier & 4 \\
Carrier separation & $0.3125 \mathrm{MHz}(=20 \mathrm{MHz} / 64)$ \\
Symbol duration & $4 \mathrm{~ms}(=$ Data $: 3.2 \mathrm{~ms}, \mathrm{CP}: 0.8 \mathrm{~ms})$ \\
CP & 16 \\
Modulation & PSPM, PSSK, QAPM, PSK, QAM \\
& $(16$-ary, 32-ary) \\
Transmit time & 1 sec (250,000 symbol) \\
Channel & Pedestrian A \\
& Vehicular A \\
\hline
\end{tabular}

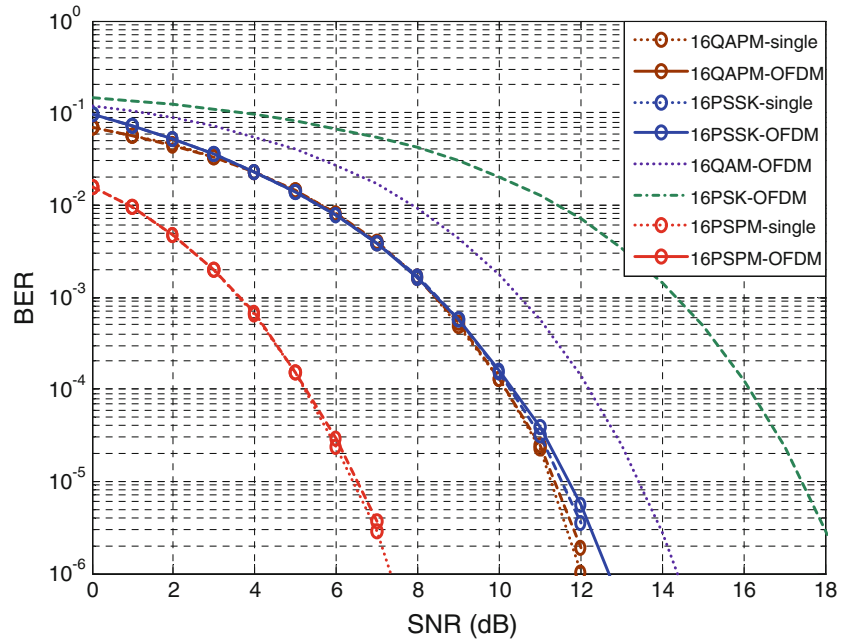

(a) BER performance of 16-ary modulation.

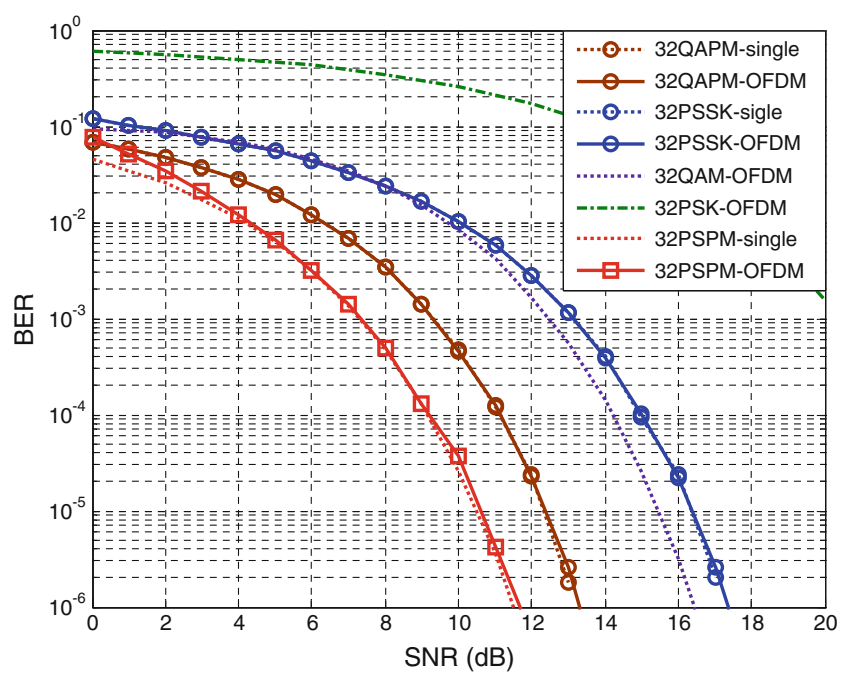

(b) BER performance of 32-ary modulation.

Fig. 11 BER performance in AWGN channel

path channel. The results for the Pedestrian A channel model are the same as those for the AWGN channel. Figure 12(a) shows the results of 16-ary modulation in a multi-path channel. The results of the Pedestrian A model are $5 \sim 6 \mathrm{~dB}$ better than those of the Vehicular A model because the channel model delay time is longer than the CP. The BER performance shows the same characteristics. Figure 12(b) shows the results of 32-ary modulation in the multi-path channel. A 32-ary PSPM has the best BER performance.

Figure 13 shows the results of a throughput simulation of each modulation scheme in the multi-path channel. Figure 13(a) shows the results of 16-ary modulation; the throughput of a 16-ary PSPM requires half the bandwidth of a 16-ary PSSK or a 16-ary QAPM. Figure 13(b) shows 
Table 3 Power efficiency and bandwidth efficiency (Bandwidth $=20 \mathrm{MHz}$ )

\begin{tabular}{|c|c|c|c|c|c|c|c|c|c|c|}
\hline \multirow[t]{2}{*}{ Modulation level } & \multicolumn{2}{|c|}{ PSPM } & \multicolumn{2}{|c|}{ PSSK } & \multicolumn{2}{|c|}{ QAPM } & \multicolumn{2}{|l|}{ PSK } & \multicolumn{2}{|c|}{ QAM } \\
\hline & 16 & 32 & 16 & 32 & 16 & 32 & 16 & 32 & 16 & 32 \\
\hline Bit rate (Mbps) & 10 & 12.5 & 20 & 25 & 20 & 25 & 40 & 50 & 40 & 50 \\
\hline Symbol Period (ns) & 50 & 50 & 50 & 50 & 50 & 50 & 50 & 50 & 50 & 50 \\
\hline Pulse-on duration (ns) & 12.5 & 12.5 & 25 & 25 & 25 & 25 & 50 & 50 & 50 & 50 \\
\hline Duty gain (dB) & 6 & 6 & 3 & 3 & 3 & 3 & 0 & 0 & 0 & 0 \\
\hline $\mathrm{Eb} / \mathrm{N} 0\left(10^{\wedge}-6\right)$ & 7.2 & 11.3 & 12.5 & 18.3 & 12 & 13.5 & 18.3 & 23 & 14.3 & 16.3 \\
\hline
\end{tabular}

the results of 32-ary modulation. The throughput of a 32ary PSPM is half that of a 32-ary PSSK with a high signalto-noise ratio (SNR). However, the throughput of a 32-ary PSPM in the Vehicular A channel model is better than that of a 32-ary PSSK at an SNR of $0 \sim 1 \mathrm{~dB}$ because PSPM modulation is less affected by the channel.

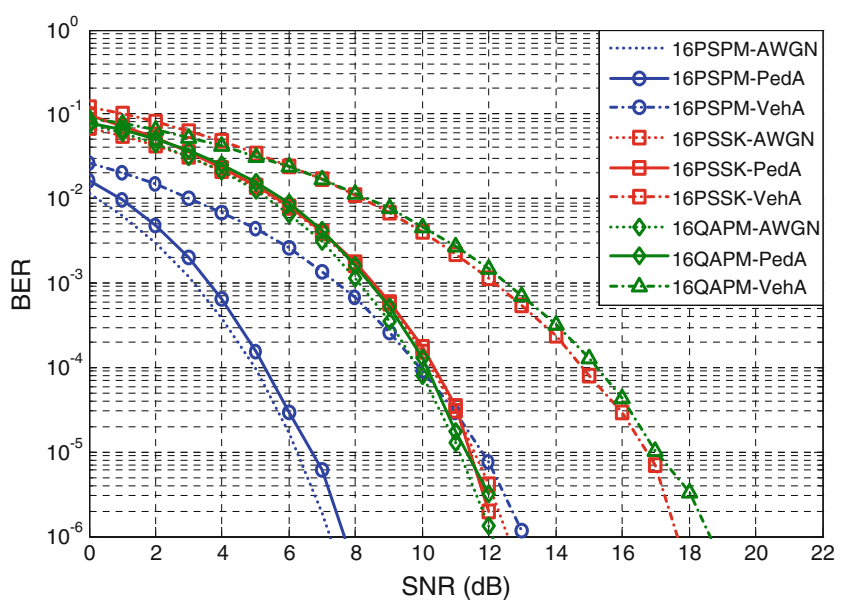

(a) BER performance of 16-ary modulation.

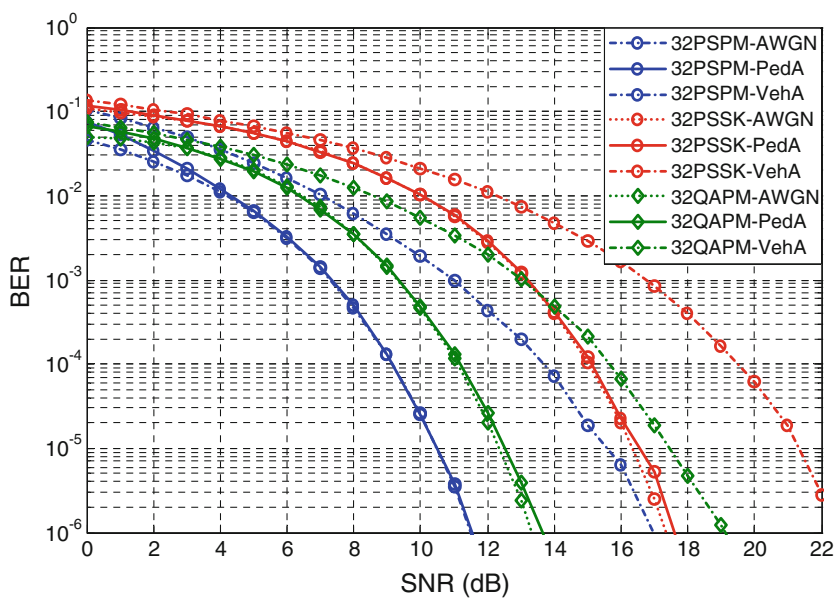

(b) BER performance of 32-ary modulation.

Fig. 12 BER performance in multi-path channel

\section{Conclusions}

In this paper, we propose a QAPM method combined with OFDM and analyze the performance of low-power-

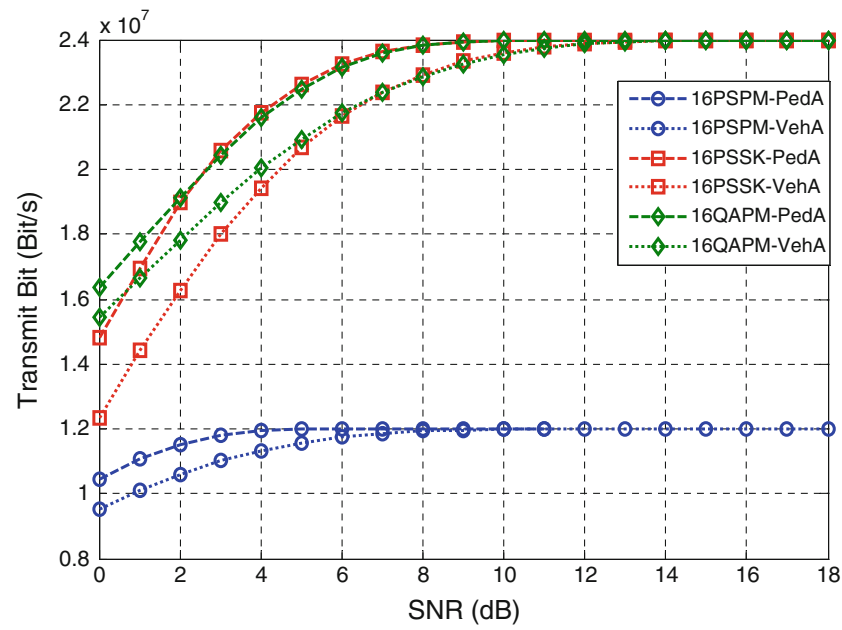

(a) Throughput performance of 16-ary modulation.

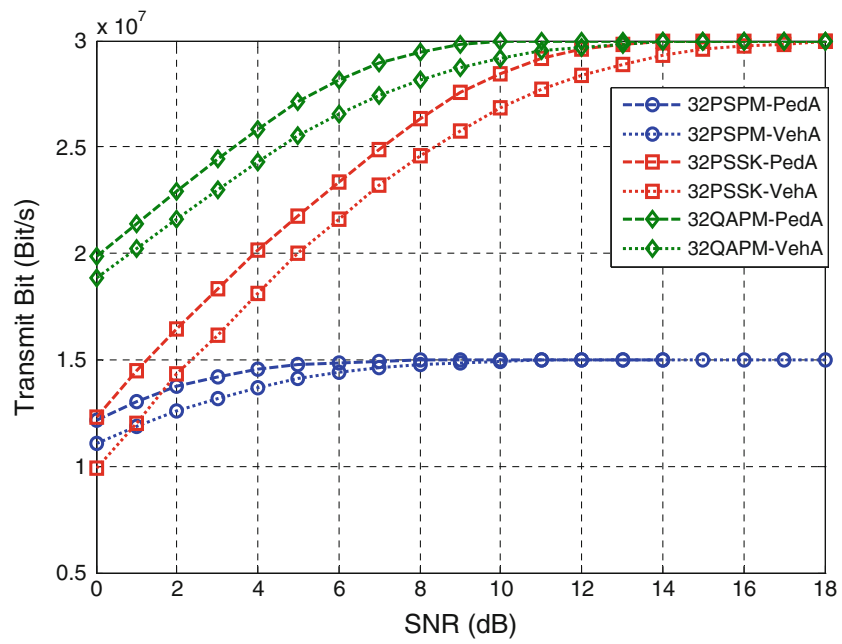

(b) Throughput performance of 32-ary modulation.

Fig. 13 Throughput performance in multi-path channel 
consumption modulation schemes such as PSPM and PSSK using OFDM transmission in a multi-path channel. In an AWGN channel, the results of low-power-consumption modulation show the same characteristics as a single-carrier system. Likewise, the simulation results of the Pedestrian A model show similar performance to that of an AWGN cannel. However, the BER performance and throughput performance deteriorate in the Vehicular A channel model because the delay time for this model is longer than the CP. The throughput of PSPM modulation is half that of PSSK and QAPM, but PSPM modulation is less affected by the channel. And we can see that throughput of QAPM modulation is better than PSSK. Therefore we can design the low power consumption transmission system using PSPM and QAPM to meet the system requirements. And we can know that a QAPM scheme could be identified as a more good a modulation scheme than the PSSK scheme.

Acknowledgement This research was supported by Basic Science Research Program through the National Research Foundation of Korea (NRF) funded by the Ministry of Education, Science and Technology (No. 2010-0007567)

Open Access This article is distributed under the terms of the Creative Commons Attribution Noncommercial License which permits any noncommercial use, distribution, and reproduction in any medium, provided the original author(s) and source are credited.

\section{References}

1. Chen M, Gonzalez S, Vasilakos A, Cao H, Leung V (2011) Body area networks: a survey, ACM/Springer Mobile Networks and Applications (MONET), April 2011

2. Long C, Reddy S, Pamarti S, He L, Karnik T (2006) Powerefficient pulse width modulation DC/DC converters with zero voltage switching control. In: Proc. Low Power Electronics and Design, ISLPED’06., pp. 326-329, Oct. 2006

3. Cho SH, Chadrakasan AP (2004) A 6.5-GHz energy efficient BFSK modulator for wireless sensor applications. IEEE J SolidState Circuit 39:731-739

4. Kim DK, Lee HS (2009) Phase-silence-shift-keying for powerefficient modulator. IEICE Trans Communication, Vol. E92-B, No.6, June. 2009

5. Oh JY, Kim JH, Lee HS (2010) PSSK modulation scheme for high data rate implantable medical device. IEEE Trans Information Technology Biomedicine 14:634-640

6. Choi JH, Ryu HG (2011) A QAPM (Quadrature Amplitude Position Modulation) for low power consumption communication. Wireless Pervasive Computing, 2011. ISWPC'2011., Feb. 2011

7. Choi JH, Ryu HG (2011) Design of QAPM modulation system with high power efficiency and SIMULINK evaluation. Advanced Communication Technology, 2011. ICACT'2011., Feb. 2011

8. 3GPP TSGR1-01-0030, "Further results on CPICH Interference Cancellation as A means for Increasing DL Capacity", Intel Corporation, Jan. 2001

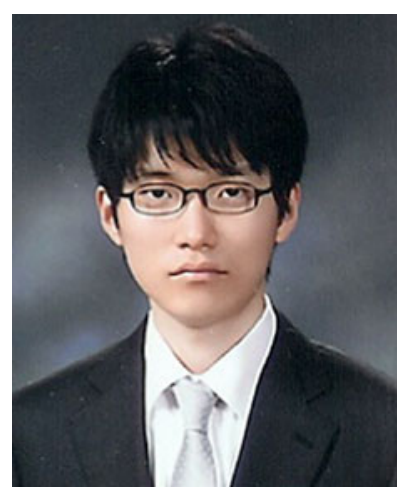

Jae-Hoon Choi was born in Dae-Jun, Republic of Korea, in 1985. He received the B.S. degree in the department of electronics, Chungbuk National University in August 2003 and 2010, respectively. $\mathrm{He}$ is currently working toward M.S degree at the Department of Electronic Engineering, Chungbuk National University, Repurblic of Korea. He research interests include digital communication system, wireless body area network and in-body communication system.

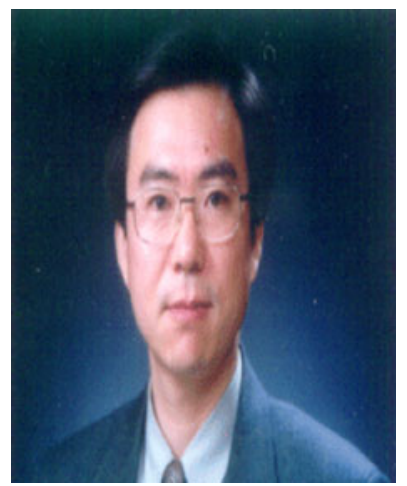

Heung-Gyoon Ryu (M'88) was born in Seoul, Republic of Korea in 1959. He received the B.S. and M.S. and Ph.D. degrees in electronic engineering from Seoul National University in 1982, 1984 and 1989. Since 1988, he has been with Chungbuk National University, Korea, where he is currently Professor of Department of Electrical, Electronic and Computer Engineering in Chungbuk National University. And he worked as director of RICIC (research institute of computer, information communication center) in Chungbuk National University from March 2002 to Feb 2004. His main research interests are digital communication systems, communication circuit design and communication signal processing.

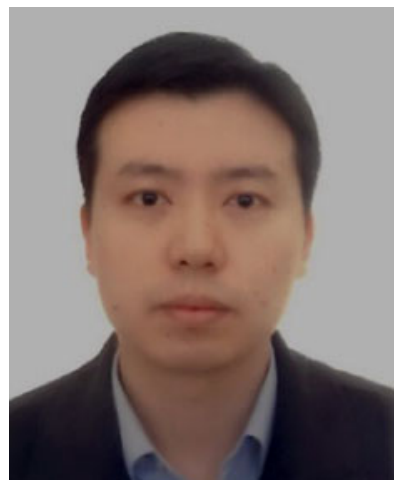

Xuedong Liang is a Postdoctoral Research Fellow at the Department of Electrical and Computer Engineering, the University of British Columbia. He received his $\mathrm{Ph} . \mathrm{D}$ degree in the Department of Informatics, University of Oslo in 2009. His research interests are in the areas of wireless communication protocols, cooperative communications, game theory and its applications, resource allocation and optimization, QoS provisioning, formal modeling and validation of wireless networks. 